\title{
Effects of rasagiline on freezing of gait in Parkinson's disease - an open-label, multicenter study
}

\author{
František Cibulcika, Jan Benetin ${ }^{\mathrm{a}}$, Egon Kurca ${ }^{\mathrm{b}}$, Milan Grofik ${ }^{\mathrm{b}}$, Miloslav Dvorakc, Denis Richter ${ }^{\mathrm{d}}$, Vladimir Donathe, \\ Jan Kothaje, Michal Minarf, Peter Valkovic ${ }^{f}$
}

\begin{abstract}
Aims. Freezing of gait is a disabling symptom in advanced Parkinson's disease. Positive effects have been suggested with MAO-B inhibitors. We report on an open label clinical study on the efficacy of rasagiline as add-on therapy on freezing of gait and quality of life in patients with Parkinson's disease.

Methods. Forty two patients with freezing of gait were treated with $1 \mathrm{mg}$ rasagiline daily as an add-on therapy. Patients were assessed at baseline and after 1,2 and 3 months of treatment. Freezing of gait severity was assessed using the Freezing of Gait Questionnaire, motor impairment by the modified MDS UPDRS part III, and quality of life using the PDQ-39 questionnaire.

Results. Patients treated with rasagiline had a statistically significant decrease in FoG-Q score and modified MDS UPDRS score after 1,2 and 3 months of therapy. A moderately strong $(r=0.686, P=0.002)$ correlation between the effects on mobility and freezing of gait was found. We also observed a statistically significant improvement in global QoL and in the subscales mobility, ADL, stigma and bodily discomfort in patients after 3 months of rasagiline therapy. A significant correlation ( $r=0.570, P=0.02$ ) between baseline FoG-Q score and the baseline score for the PDQ Mobility subscale was found.
\end{abstract}

Conclusion. In our study rasagiline as add-on antiparkinsonian therapy significantly improved mobility, freezing of gait and quality of life. The positive effect on freezing of gait appears to be related to improvement of mobility.

Key words: Parkinson's disease, freezing of gait, Rasagiline

Received: February 22, 2016; Accepted: April 13, 2016; Available online: April 21, 2016

http://dx.doi.org/10.5507/bp.2016.023

${ }^{a}$ Department of Neurology, Faculty of Medicine, Slovak Medical University in Bratislava and University Hospital Bratislava, Slovak Republic ${ }^{b}$ Department of Neurology, Jessenius Faculty of Medicine, Comenius University in Bratislava, Martin and University Hospital Martin, Slovak Republic

'Department of Neurology, Hospital Spisska Nova Ves, Slovak Republic

${ }^{d}$ Department of Neurology, University Hospital Bratislava, Slovak Republic

${ }^{e}$ Department of Neurology, Slovak Health University, Roosevelt Hospital Banska Bystrica, Slovak Republic

fSecond Department of Neurology, Faculty of Medicine, Comenius University in Bratislava, Slovak Republic

Corresponding author: Frantisek Cibulcik, e-mail: cibulcik@hotmail.com

\section{INTRODUCTION}

Freezing of gait (FoG) is a gait disorder that occurs in patients with Parkinson's disease (PD). Clinically, FoG is defined as a few seconds episodic failure to produce effective steps in the absence of evident causative factors other than PD and "high-level" gait disorder. FoG is clinically important as it can cause loss of balance and is the most frequent cause of falls in PD patients. The pathophysiology of FoG is complex, and some authors consider it a cardinal symptom of PD, whilst others have suggested links with high-dose levodopa, treatment with dopamine agonists, or the duration of the disease ${ }^{1}$.

Effective treatment of FoG is one of the greatest challenges in the management of PD, and there is no generally accepted approach. Sensory tricks and rhythmic sonic perceptions have been effective, and positive effects of deep brain stimulation in the area of the subthalamic nucleus and pedunculopontine nucleus have been reported. Many drug treatments have been tried for FoG, and these were reviewed by Giladi in 2008 (ref. ${ }^{1}$ ). Rasagiline - a monoamine oxidase B inhibitor used in the treatment of PD as monotherapy or as adjunct therapy, shows most consistent effects. The Slovak Republic was one of the first EU countries to launch rasagiline for the treatment of PD. Our anecdotal experience suggested a positive effect of rasagiline on FoG. We therefore decided to conduct a formal study of the effects of rasagiline in everyday clinical practice, focusing on FoG.

\section{METHODS}

\section{Patients}

Between April and December 2011, we enrolled patients with the clinical diagnosis of PD according to United Kingdom Parkinson's Disease Society Brain Bank and ICD-10 criteria in this open clinical trial at 6 outpatient Slovak movement disorders centers. Patients with symptoms of FoG, defined as having scored 1 or more for Question 3 of the Freezing of Gait Questionnaire (FoG-Q) - " Do you feel that your feet get glued to the 
floor while walking, making a turn or when trying to initiate walking (freezing)?" (ref. ${ }^{2}$ ). Patients with more severe motor disorders, psychiatric illnesses, neuromuscular disorders, symptomatic orthostatic hypotension, or prominent "off" dyskinesias were excluded. Treatment of PD must have been unchanged for at least 6 weeks before study enrolment, and treatment with selegiline was not allowed 2 months prior to enrolment.

The study was approved by the local ethics committee, and informed consent was obtained from all the patients before enrolment.

\section{Variables and methods of evaluation}

Basic demographic characteristics of each patient were collected. We used the FoG-Q proposed by Giladi et al. to assess the signs of FoG as primary outcome ${ }^{2}$. Motor impairment as a secondary outcome was evaluated by means of the Slovak translation of the MDS revised Unified Parkinson's Disease Rating Scale (MDS UPDRS part III, items 3.3 - 3.18) ( ref. $^{3}$ ), and the stage of disease by the Hoehn and Yahr Scale. We evaluated as another secondary outcome the quality of life (QoL) with the Slovak version of the PDQ-39 (ref., ${ }^{4,5}$ ). This questionnaire is currently the only validated tool for the evaluation of FoG. Evaluations were done at baseline and after 1, 2 and 3 months.

\section{Treatment}

In this study, rasagiline was administered as an adjunct therapy at a single daily dose of $1 \mathrm{mg}$ in the morning for
3 months. Changes to the patient's existing antiparkinsonian regimen were not permitted. Standard adverse event reporting procedures for clinical trials were followed.

\section{Statistical analyses}

All findings were presented as means \pm standard deviations. Changes of continuous variables in time were evaluated using ANOVA with repeated measures. The Greenhouse-Geisser correction was used to adjust for nonsphericity for the overall F-tests. Pairwise comparisons were performed with Bonferroni correction for the 95\% confidence intervals.

In addition, the Spearman correlation test was used to analyze relationships between possible contributory factors and the defined endpoints. $P$ values $<0.05$ were considered statistically significant. All statistical analyses were conducted using commercially available software (SPSS for Windows, version 19.0).

\section{RESULTS}

Forty two patients were enrolled and treated; 2 failed to complete the study: one because of worsening peak-ofdose dyskinesia, and one because of new onset of night hallucinations. Since all the failures were in the first week of therapy, we excluded these patients from further data analyses. The final analysis data set thus consists of 40 patients ( 22 male and 18 female ) - 10 in Hoehn and Yahr stage 2, 23 in stage 3 and seven in stage 4 . The mean age

Table 1. UPDRS III and FoG-Q changes.

\begin{tabular}{lccccccc}
\hline & \multicolumn{3}{c}{ Timepoint } & \multicolumn{3}{c}{ Change from baseline (95\% CI) } \\
& \multirow{2}{*}{ Baseline } & Month 1 & Month 2 & Month 3 & Month 1 & Month 2 & Month 3 \\
\hline UPDRS III & $40.4 \pm 14.9$ & $37.2 \pm 14.2^{*}$ & $36.7 \pm 14.3^{* *}$ & $36.1 \pm 15.0^{* * *}$ & $-3.2 \pm 7,4$ & $-3.7 \pm 6.9$ & $-4.4 \pm 5.5$ \\
& & & & & $(-5.5 ;-0.8)$ & $(-5.9 ;-1.4)$ & $(-6.1 ;-2.6)$ \\
FoG-Q & $12.6 \pm 4.2$ & \multirow{2}{*}{$11.1 \pm 4.7^{* *}$} & $10.4 \pm 4.7^{* * *}$ & $9.9 \pm 4.8^{* * *}$ & $-1.6 \pm 3.7$ & $-2.3 \pm 3.4$ & $-2.7 \pm 3.3$ \\
& & & & & $(-2.8 ;-0,4)$ & $(-3.3 ;-1.2)$ & $(-3.8 ;-1.7)$ \\
\hline
\end{tabular}

UPDRS, Unified Parkinson Disease Rating Scale; FoG-Q, Freezing of Gait Questionnaire; ${ }^{*} P<0.05 ;{ }^{* *} P<0.01 ;{ }^{* * *} P<0.001 ; P$ values and $95 \%$ confidence intervals - repeated measures ANOVA

Table 2. PDQ-39 changes.

\begin{tabular}{|c|c|c|c|c|}
\hline & Baseline & Month 3 & Change $(95 \% \mathrm{CI})$ & $P$ \\
\hline PDQ MOB & $45.6 \pm 22.9$ & $41.2 \pm 24.6$ & $-4.4(-7.3 ;-1,4)$ & $<0.001$ \\
\hline PDQ ADL & $47.6 \pm 22.0$ & $44.8 \pm 21.9$ & $-3.0(-6.1 ; 0.2)$ & 0.063 \\
\hline PDQ EMO & $21.8 \pm 14.5$ & $19.5 \pm 14.5$ & $-2.0(-4.6 ; 0.7)$ & 0.099 \\
\hline PDQ STIG & $34.3 \pm 24.5$ & $27.5 \pm 25.3$ & $-6.8(-11.0 ;-2.6)$ & 0.007 \\
\hline PDQ SOC & $7.6 \pm 18.3$ & $6.2 \pm 15.1$ & $-1.1(-3.0 ; 0.7)$ & 0.199 \\
\hline PDQ COG & $15.4 \pm 11.8$ & $15.7 \pm 12.0$ & $-0.3(-3.0 ; 2.3)$ & 0.834 \\
\hline PDQ COM & $14.4 \pm 16.6$ & $14.4 \pm 16.1$ & $-0.2(-3.8 ; 3.4)$ & 0.988 \\
\hline PDQ BODY & $27.5 \pm 17.3$ & $23.4 \pm 18.9$ & $-4.2(-8.0 ;-0.3)$ & 0.039 \\
\hline PDQ-39 Total & $31.4 \pm 13.2$ & $28.7 \pm 14.7$ & $-2.7(-4.4 ;-1.0)$ & $<0.001$ \\
\hline
\end{tabular}

PDQ MOB, PDQ Mobility; PDQ ADL, PDQ Activities of Daily Living; PDQ EMO, PDQ Emotional Wellbeing; PDQ STIG, PDQ Stigma; PDQ SOC, PDQ Social Support; PDQ COG, PDQ Cognition; PDQ COM, PDQ Communication; PDQ BODY, PDQ Bodily Discomfort; $P$ values and $95 \%$ confidence intervals - repeated measures ANOVA 
was $69.5 \pm 7.9$ years, the mean duration of Parkinson's disease was $8.3 \pm 4.3$ years and mean duration of FoG was $3.3 \pm 1.8$ years.

A repeated measures ANOVA showed that there was a significant $(P<0.001)$ time effect on the change of UPDRS III. The mean modified MDS UPDRS III scale score at baseline was $40.4 \pm 14.9$. Motor function improved statistically significantly during treatment: the mean score after 1 month was $37.2 \pm 14.2(P<0.05)$, after 2 months $36.7 \pm 14.3(P<0.01)$, and at the end of the study $36.1 \pm 15.0(P<0.001)$. Change of UPDRS III score at month 3 was $-4.4(95 \% \mathrm{CI}=-6.1$ to -2.6$)$.

A repeated measures ANOVA showed that there was a significant $(P<0.001)$ time effect on the change of FoG $(P<0.001)$. The mean FoG-Q score at baseline was 12.6 \pm 4.2 and improved statistically significantly to $11.1 \pm 4.7$ after 1 month $(P<0.01), 10.4 \pm 4.7(P<0.001)$ after 2 months, and $9.9 \pm 4.8(P<0.001)$ at the end of the study. The mean change of FoG at month 3 was $-2.7(95 \%$ $\mathrm{CI}=-3.8$ to -1.7 ) (Table 1$)$. No statistically significant correlation was found between the changes in UPDRS III or FoG-Q scores after 1, 2 or 3 months of therapy and baseline scores for age, duration of PD, or stage of FoG. However, we found a significant correlation between the changes in UPDRS III and FoG-Q scores $(r=0.686, P$ $=0.002$ ).

QoL improved statistically significantly $(P<0.001)$ with the PDQ-39 total mean scores of $31.4 \pm 13.2$ at baseline and $28.7 \pm 14.5$ after 3 months. Statistically significant improvements were also observed for the mobility subscales: Mobility (MOB) $45.6 \pm 22.9$ versus $41.2 \pm 24.6$; $P<0.001$; Stigma (STIG) $34.3 \pm 24.5$ versus $27.5 \pm 25.3$; $P=0.003$; Activities of daily life (ADL) $47.6 \pm 22.0$ versus $44.8 \pm 21.9 ; P=0.007$; and Bodily discomfort (BODY) $27.5 \pm 17.3$ versus $23.4 \pm 18.9 ; P=0.039$ (Table 2 ).

The study with 40 patients does not have enough power and control of bias to study the impact of baseline characteristics on outcomes. In an exploratory analysis, statistically significant (weak/moderate) correlations between baseline FoG and changes in QoL after 3 months were found between duration of FoG and the score on the PDQ subscale Communication ( $\mathrm{r}=0.460, P=0.047$ ), between total FoG-Q score and PDQ-39 total score ( $\mathrm{r}=$ $0.490, P=0.041)$ and the score on the PDQ subscale Mobility ( $\mathrm{r}=0.570, P=0.02)$.

\section{DISCUSSION}

The effect of rasagiline on FoG has been reported in case reports, and open, observational, and placebo-controlled double-blind studies. Coria and Cozar-Santiago ${ }^{6}$ described an 84-year-old patient who developed parkinsonian gait, fear of falling, and frequent freezing episodes as part of high-level gait disorder. Treatment with $1 \mathrm{mg}$ rasagiline daily led to a long-lasting improvement in freezing episodes. Levin and colleagues ${ }^{7}$ conducted an open observational 3-month study of efficacy and safety of rasagiline in 45 non-demented, advanced PD patients with motor fluctuations on long-term levodopa therapy (the
'AZIMUT' study). All patients received $1 \mathrm{mg}$ rasagiline once daily as an adjunct to stable antiparkinson therapy. At the end of the third month, the UPDRS III score ("on" state) had decreased by $10 \%$. The severity of FoG measured as an item on the dyskinesia and fluctuation scale had decreased by $15 \%$. The authors suggested that rasagiline appeared to be more clinically effective in patients with more severe FoG before treatment.

In the LARGO study ${ }^{8}$, rasagiline treatment resulted in a significant improvement compared to placebo in UPDRS - Postural Instability/Gait Difficulty (PIGD) score $(-0.31$ difference; $P=0.034)$ and UPDRS freezing score $(-0.16$ difference; $P=0.045)$. These scores, although not fully validated, rate symptoms such as postural instability and gait problems. Unfortunately, the analyses were post hoc and no correlation analysis was done. A subgroup of 454 PD patients from the LARGO study was tested for the effect of rasagiline in comparison with entacapone and placebo on freezing severity after 10 weeks of treatment. The total FoG-Q score improved by 1.17 points under rasagiline $(P=0.045$ versus placebo $), 1.11$ under entacapone ( $P=0.066$ versus placebo $)$, and by 0.48 points under placebo ${ }^{9}$. Only a weak correlation was found between the effects on total daily "off" duration and the FoG-Q score in the rasagiline, entacapone and placebo treatment arms. Rasagiline statistically significantly reduced the severity of FoG by 0.94 points versus placebo $(P=0.02)$ in patients with mild to moderate FoG $(\leq 15)$, while patients with severe FoG at baseline did not show any significant benefit. Despite a statistically significantly superior effect of rasagiline and entacapone over placebo, the clinical significance of a one-point improvement in the FoG-Q is not clear. In our study, we observed a statistically significant improvement in motor score on the modified MDS UPDRS III scale. This improvement was statistically significant as early as 1 month after the start of rasagiline and continued throughout the second and third month of treatment. Our findings were therefore similar to those of the AZIMUT study (10.6\% improvement after 3 months in our study; 10\% improvement on the UPDRS III measured in "on" state in the AZIMUT study), and also the LARGO study (improvement by $12.3 \%$ on the motor subscale of the UPDRS scale after 16 weeks of treatment).

Our findings also confirmed the efficacy of rasagiline on motor symptoms of PD in an add-on dosing regimen. After adding rasagiline in our study, we observed a statistically significant improvement in FoG symptoms as early as after 1 month of treatment and this was still present after 2 and 3 months of treatment. After 3 months of treatment, the 2.7 point improvement in our patients was more marked than that described by Giladi et al. (2004) of 1.17 points after 10 weeks of treatment. The difference might - at least in part - be explained by the open design of our study and different inclusion criteria. We included only patients with FoG present at baseline, whereas this was the case for only $86 \%$ of patients in Giladi et al.'s study. A further substantial difference is that we found a moderately strong correlation between the improvement in motor score and improvement in FoG score. Giladi 
et al. did not, and they suggested this could be due to a non-dopaminergic mechanism of action on freezing by rasagiline. By contrast, our results suggest a possible relationship between improvement in dopaminergic stimulation and improvement in FoG in patients with PD.

We know from the literature that idiopathic PD has a major negative impact on the quality of life measured by PDQ-39. Among 11 mobility-related symptoms, difficulty in turning, festination, freezing and shuffling of gait had the most significant effect on QoL scores ${ }^{10}$. It is possible that a therapeutic effect on these motor symptoms might positively influence QoL in PD patients. Martinez and Deusch $1^{11}$ reviewed the literature and found 61 clinical trials of patients with PD that had QoL as a planned outcome parameter. They found that only unilateral pallidotomy, deep brain stimulation of the subthalamic nucleus, and rasagiline improved QoL in PD patients. In a placebo-controlled study in early PD (ref. ${ }^{12}$ ), rasagiline monotherapy resulted in a significant improvement in self-image/sexuality domain subscore and global QoL measured on the PD-QUALIF. In our study in PD patients with FoG on add-on therapy with rasagiline, we observed an improvement in global PDQ-39 score and the scores of three subscales, with a particularly marked improvement for the mobility subscale. It therefore seems that rasagiline improves QoL in PD patients with FoG, especially by improving mobility parameters.

\section{CONCLUSION}

Rasagiline added to antiparkinsonian therapy in patients with freezing of gait significantly improved mobility, freezing of gait and quality of life. The effect on freezing of gait appears to be related to dopaminergic action on mobility.

Acknowledgement: Lundbeck Slovakia sponsored the study and data analysis.

Author contributions: FC: study design, data collection, data processing and interpretation, literature search, manuscript writing, JB, EK, PV: study design, data collection, data processing and interpretation, manuscript writing,
MG, MD, DR, VD, JK, MM: study design, data collection, data processing and interpretation.

Conflict of interest statement: The authors state that there are no conflicts of interest regarding the publication of this article.

\section{REFERENCES}

1. Giladi N. Medical treatment of freezing of gait. Mov Disord 2008;23:S482-S488

2. Giladi N, Shabtai H, Simon ES, Biran S, Tal J, Korczyn AD. Construction of freezing of gait questionnaire for patients with Parkinsonism. Parkinsonism Relat Disord 2000;6:165-70.

3. Goetz CG, Fahn S, Martinez-Martin P, Poewe W, Sampaio C, Stebbins GT, Stern MB, Tilley BC, Dodel R, Dubois B, Holloway R, Jankovic J, Kulisevsky J, Lang AE, Lees A, Leurgans S, LeWitt PA, Nyenhuis D, Olanow CW, Rascol O, Schrag A, Teresi JA, Van Hilten JJ, LaPelle N. Movement disorder society-sponsored revision of the Unified Parkinson's disease rating scale (MDS-UPDRS): process, format, and clinimetric testing plan. Mov Disord 2007;22:41-7.

4. Peto V, Jenkinson C, Fitzpatrick R, Greenhall R. The development and validation of a short measure of functioning and well-being for individuals with Parkinson's disease. Qual Life Res 1995;4:241-8.

5. Martinez-Martin $P$, Jeukens-Visser M, Lyons KE, Rodriguez-Blazquez C, Selai C, Siderowf A et al. Health-related quality-of-life scales in Parkinson's disease: critique and recommendations. Mov Disord 2011;26:2371-80

6. Coria F, Cozar-Santiago MP: Rasagiline improves freezing in patient with primary progressive freezing gait. Mov Disord 2008;23:449-51.

7. Levin OS, Boiko AN, Ivanov AK. Efficacy of rasagiline in patients with advanced Parkinson's disease with motor fluctuation (Azimut study). Zh Nevrol Psikhiatr Im S S Korsakova 2010;110:42-7.

8. Rascol 0, Brooks DJ, Melamed E, Oertel W, Poewe W, Stocchi F, Tolosa E. Rasagiline as an adjunct to levodopa in patients with Parkinson's disease and motor fluctuations (LARGO, Lasting effect in Adjunct therapy with Rasagiline Given Once Daily): a randomised, doubleblind, parallel-group trial. Lancet 2005;365:947-54.

9. Giladi N, Rascol O, Melamed E, Oertel W, Poewe W, Stocchi F, Tolosa E. Rasagiline treatment can improve freezing of gait in advanced Parkinson's disease: A prospective, randomised, double blind, placebo and entacapone controlled study. Mov Disord 2004;19:S191-2.

10. Rahman S, Griffin HJ, Quinn NP, Jahanshahi M. Quality of life in Parkinson's disease: the relative importance of the symptoms. Mov Disord 2008;23:1428-34.

11. Martinez-Martin P, Deuschl G. Effect of medical and surgical interventions on health-related quality of life in Parkinson's disease. Mov Disord 2007;22:757-65.

12. Biglan KM, Schwid S, Eberly S, Blindauer K, Fahn S, GorenT, Kieburtz K, Oakes D, Plumb S, Siderowf A, Stern M, Shoulson I; Parkinson Study Group. Rasagiline improves quality of life in patients with early Parkinson's disease. Mov Disord 2006;21:616-23. 